\title{
A Comparison of Phase Estimation Between Hall Sensors and Sliding Mode Observer
}

\author{
Ziyou Song ${ }^{1, \mathrm{a}}$, Jianqiu $\mathrm{Li}^{1, \mathrm{~b}}$, Minggao Ouyang ${ }^{1, \mathrm{c}}$, Liangfei $\mathrm{Xu}^{1, \mathrm{~d}}$ \\ ${ }^{1}$ State Key Laboratory of Automotive Safety and Energy, Tsinghua University, Beijing, China \\ aziyou.songthu@gmail.com, blijianqiu@tsinghua.edu.cn, couymg@tsinghua.edu.cn, \\ dxuliangfei@tsinghua.edu.cn
}

Keywords: phase estimation, Hall sensors, sliding mode observer, starting state, steady state, speed fluctuation state.

\begin{abstract}
This paper presents a comparison about phase estimation between Hall sensors and sliding mode observer (SMO). The phase estimation result corresponds to the control effect of Permanent-magnet Synchronous Motor (PMSM). Low-resolution Hall-effect sensor is widely used in PMSM because of its good comprehensive performance. Sliding mode observer is a frequently-used algorithm of PMSM sensorless control. The rotor phase estimated by Hall sensors has a good steady-state performance but some ripple or chattering components occur when the rotor is accelerating, decelerating or fluctuating. As a contrast, the phase estimated by SMO has an attractive robustness to speed fluctuation as well as parameter variations but the estimation result has some vibrations under steady condition which is caused by the SMO algorithm itself. This paper contrasts the SMO with Hall sensors in view of control effects both under starting state, steady state and speed fluctuation state. The comparison is illustrated by the phase estimation results and the phase currents response. All the work is realized by Matlab/Simulink and the simulation results show the relative merits of SMO and Hall sensors.
\end{abstract}

\section{Introduction}

Permanent-magnet synchronous motors (PMSM) are widely used for many engineering applications because of their high torque, high power density and high efficiency [1]. With regard to the PMSM control, the field-oriented control algorithm is widely used relying on its legible logic and good performance on ripple-free torque production [2,3]. Low-resolution Hall-effect sensor is popularly used to estimate the rotor position $[4,5]$ but it has several disadvantages from the standpoint of cost, reliability and encumbrance [6]. An increasing number of Hall sensors will bring a better resolution of the estimated rotor position. Generally three Hall sensors are used in one PMSM to make sure the estimation precision. Meanwhile, the sensorless control strategies become more and more popular because of their low-cost and high reliability and two kinds of them seem to be preferable: signal injection techniques [7] and state observers. The sliding mode observer has been proposed for a long time and is widely used in sensorless control of PMSM because that it has a good robustness to rotor speed fluctuation and parameter variations $[8,9,10]$. But the SMO cannot start the PMSM without other devices and the phase estimation result of SMO has some vibration components when contrasted to the Hall sensors.

This paper contrasts the SMO with Hall sensors concerning to the phase estimation and phase currents response both under starting state, steady steady and speed fluctuating steady. The PMSM control model is based on Matlab/Simulink and the simulation results show the comparison distinctly. This paper is organized as follows: In section II, the sliding mode observer is designed. In section III, the phase estimation method realized by Hall sensors is illustrated. In section IV, the simulation model of the PMSM drive system is presented and the comparison between SMO and Hall sensors is realized by the simulation. In section $\mathrm{V}$, the conclusions are presented and the results of the simulation are analyzed in details. 


\section{Phase Estimation Processes of SMO [8,9]}

The $\alpha-\beta$ model for PMSM in the stationary reference frame is characterized by Eq. 1 :

$i_{s}=A i_{s}+B v_{s}+K_{e} v_{i}+\xi$

where $i_{s}=\left[\begin{array}{ll}i_{\alpha} & i_{\beta}\end{array}\right]^{T}$ is the stationary $\alpha-\beta$ currents vector, $v_{s}=\left[\begin{array}{ll}v_{\alpha} & v_{\beta}\end{array}\right]^{T}$ is the stationary $\alpha-\beta$ voltages vector, $v_{i}=\left[\begin{array}{ll}-\omega \sin \theta & \omega \cos \theta\end{array}\right]^{T}$ is the back EMF vector, $A=\left(-\frac{R_{s}}{L_{s}}\right) I, B=\left(\frac{1}{L_{s}}\right) I, R_{s}$ and $L_{s}$ are stator winding resistance and inductance respectively, $\mathrm{I}$ is $2 \times 2$ identity matrix, $K_{E}$ is the EMF constant, $\xi$ $=\left[\begin{array}{ll}\xi_{\alpha} & \xi_{\beta}\end{array}\right]^{T}$ is the disturbance vector.

The sliding mode observer can be described as:

$\frac{d \hat{i}_{s}}{d t}=A \hat{i}_{s}+B v_{s}+K_{s w} \operatorname{sgn}\left(\hat{i_{s}}-i_{s}\right)$

where $\hat{i}_{s}$ is the estimated value of is, $K_{s w}=k I$ : the observer switching gain, $\operatorname{sgn}\left(\hat{i_{s}}-i_{s}\right)=\left[\operatorname{sgn}\left(\hat{i_{\alpha}}-i_{\alpha}\right) \operatorname{sgn}\left(\hat{i_{\beta}}-i_{\beta}\right)\right]^{T}$.

The sliding hyperplane $S$ is realized by the switching functions as:

$$
S=\hat{i}_{s}-i_{s}=e_{s}
$$

The estimation error dynamic is obtained by subtracting Eq. 1 from Eq. 2 as:

$\dot{e}_{s}=A e_{s}-K_{E}\left(v_{i}\right)+K_{s w} \operatorname{sgn}\left(e_{s}\right)-\xi$

To satisfy the necessary conditions for the sliding mode convergence, $K_{s w}$ must be chosen to satisfy $\dot{e}_{s} e_{s}^{T}<0$. Paper [8] gives a equivalent method, so the expression is:

$\dot{e}_{s}=e_{s}=0$

The characteristics of SMO on the sliding hyperplane might be defined as:

$$
\begin{aligned}
& K_{s w} \operatorname{sgn}\left(e_{s}\right)=K_{E}\left(v_{i}\right)+\xi \\
& K_{E}\left[\begin{array}{l}
-\omega \sin \theta \\
\omega \cos \theta
\end{array}\right]-\left[\begin{array}{l}
\xi_{\alpha} \\
\xi_{\beta}
\end{array}\right]=K_{s w}\left[\begin{array}{c}
\operatorname{sgn}\left(\hat{i_{\alpha}}-i_{\alpha}\right) \\
\operatorname{sgn}\left(\hat{i_{\beta}}-i_{\beta}\right)
\end{array}\right]
\end{aligned}
$$

Estimated back EMF is obtained by the low pass filter of the switching signals. The conventional low pass filter has a constant cutoff frequency and this kind of filter is regardless of the rotor speed. In order to fulfil the requirement of wide speed response, the low pass filter should have a variable cutoff frequency [10]. This new filter has a good performance but it has a higher demand on the estimated speed. The noise in the estimated speed impacts the low pass filter a lot. The expression of the variable cutoff frequency filter is shown by Eq. 8.

$$
\begin{aligned}
& \hat{e}_{\alpha}=\frac{k \omega}{s+k \omega} \operatorname{sgn}\left(\hat{i_{\alpha}}-i_{\alpha}\right) \\
& \hat{e}_{\beta}=\frac{k \omega}{s+k \omega} \operatorname{sgn}\left(\hat{i_{\beta}}-i_{\beta}\right)
\end{aligned}
$$

where $k$ is a constant value which depends on the characteristic of the drive system. So the estimated rotor phase can be obtained:

$$
\hat{\theta}=\hat{\theta}_{0}+\Delta \hat{\theta}=\arctan \left(-\frac{\hat{e}_{\alpha}}{\hat{e}_{\beta}}\right)+\arctan \left(\frac{1}{k}\right)
$$

where $\Delta \hat{\theta}$ is the compensation because of the phase delay caused by the low pass filter. And the SMO control system is shown in Fig. 1. 


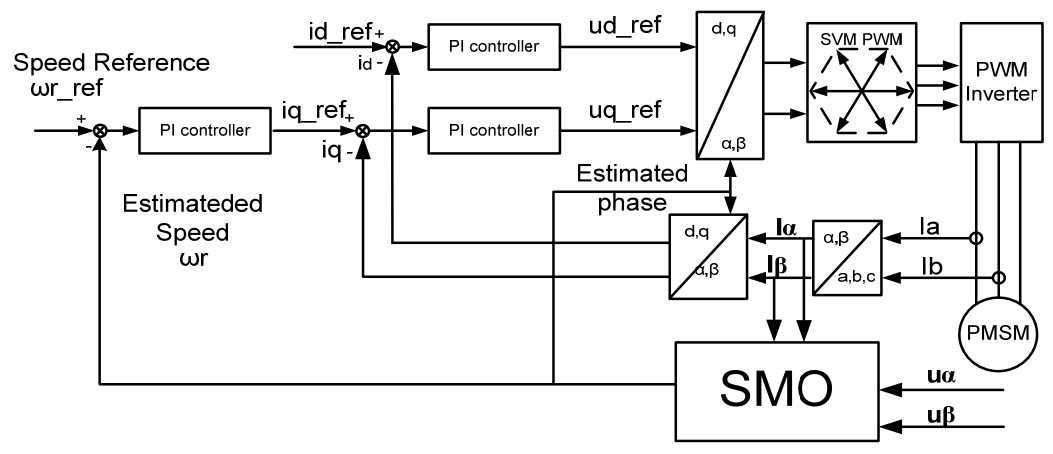

Fig. 1. The SMO sensorless control scheme.

\section{Phase Estimation Processes of Hall sensors}

In this research, three Hall sensors are used to estimate the rotor phase. One physical period (one circle of the motor) consists of 23 electric periods because of the 23 pole pairs and one electric period is divided into six different sections by the three Hall sensors. The Hall signal during one electric period is shown by Fig. 2.

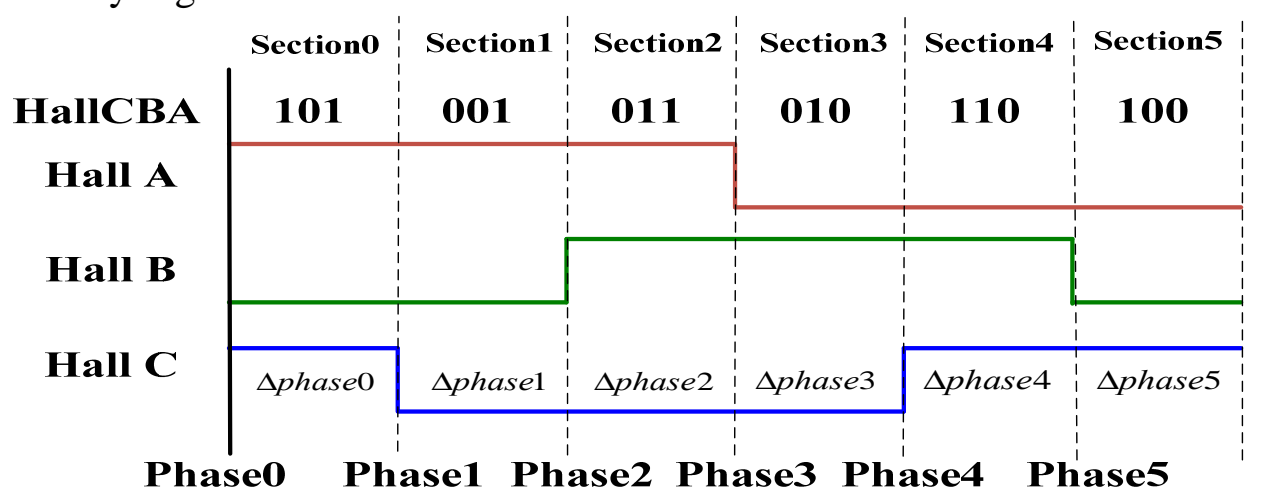

Fig. 2. Three Hall signals during one electric period.

The six sections can be encoded via reading the three Hall values (Hall_C, Hall_B, Hall_A) which are $5,1,3,2,6,4$. There are six accurate phase references at the edge of these six sections which are Phase0, Phase1, Phase2, Phase3, Phase4, Phase5. The Fig. 3 illustrates this issue more visualized.

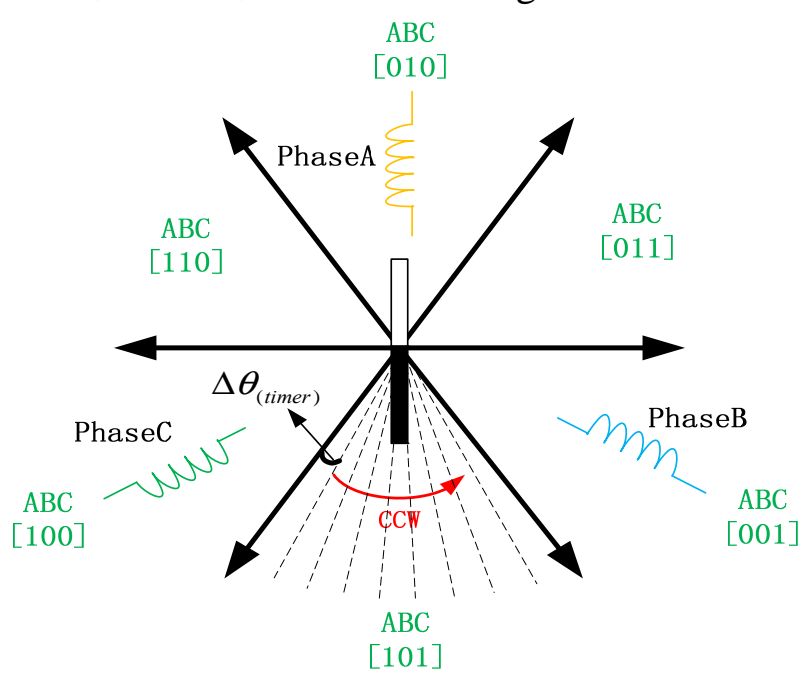

Fig. 3. Three Hall signals divide one electric period into six sections 
The six sections can be encoded via reading the three Hall values (Hall_C, Hall_B, Hall_A) which are $5,1,3,2,6,4$. There are six accurate phase references at the edge of these six sections which are Phase0, Phase1, Phase2, Phase3, Phase4, Phase5. The Fig. 3 illustrates this issue more visualized.

With the rotor rotating anticlockwise like Fig. 3 shows, the Hall value changes regularly. Any changes of Hall value could be captured by the ECU and then the ECU reads the three Hall values to judge which section edge the rotor is. For instance, when the ECU captures a Hall value changing from 101 to 001 then it is easy to know that $\theta=$ Phase1. But how to estimate the rotor phase within these six sections is an important problem which can be solved by using the timer both in pratical application and simulation. The motor rotary speed can be calculated as Eq. 10.

$$
\omega=\frac{\Delta \text { Phase }(i)}{P_{n} \times \Delta t}, i=0,1,2,3,4,5
$$

where $\omega$ is the rotor angular velocity, $\Delta t$ is the time of the rotor passing the i section, $\Delta$ Phase $(i)$ is the phase interval of the i section.

In theory, the $\Delta$ Phase $(i)$ should be 60 (electric degree). But the three Hall sensors have undefined installation error so that the $\Delta$ Phase $(i)$ are not uniform. The rotor phase increment during one timer period can be calculated by Eq. 11.

$$
\Delta \theta_{(\text {timer })}=\frac{\omega P_{n}}{f_{\text {timer }}}
$$

where $\Delta \theta_{\text {(timer) }}$ is the rotor phase increment per timer period, $f_{\text {tmier }}$ is the timer frequency.

The rotor phase is estimated by plus $\Delta \theta_{\text {(tmier) }}$ to $\theta$ every timer period and $\Delta \theta_{(\text {timer })}$ refines the sections like Fig. 3 shows.

In FOC algorithm, the rotary speed of last section is used to predict the rotor position now which implies that the speed is not the real speed at the present moment. The predicted rotor phase contains errors if the motor speed is not constant, for example, the PMSM is accelerating or decelerating. So the phase estimation result may have some deformity when the PMSM speed is fluctuating.

\section{Simulation Results and Analyses}

The comparison between Hall sensors and SMO is performed on a PMSM with the following parameters.

Table 1.The parameters of the PMSM

\begin{tabular}{cc}
\hline Parameter & Value \\
\hline Number of phases & 3 \\
Number of poles & 23 \\
Timer frequency & $10[\mathrm{us}]$ \\
Q-axis stator inductance & $8.5[\mathrm{mH}]$ \\
D-axis stator inductance & $8.5[\mathrm{mH}]$ \\
Stator phase resistance & $0.2[\mathrm{ohm}]$ \\
Torque constant & $6.0375[\mathrm{~N}-\mathrm{m} / \mathrm{A}]$ \\
Inertia & $0.1\left[\mathrm{~kg} \cdot \mathrm{m}^{2}\right]$ \\
\hline
\end{tabular}

The simulation model to simulate the PMSM drive system is described in Fig. 4. 


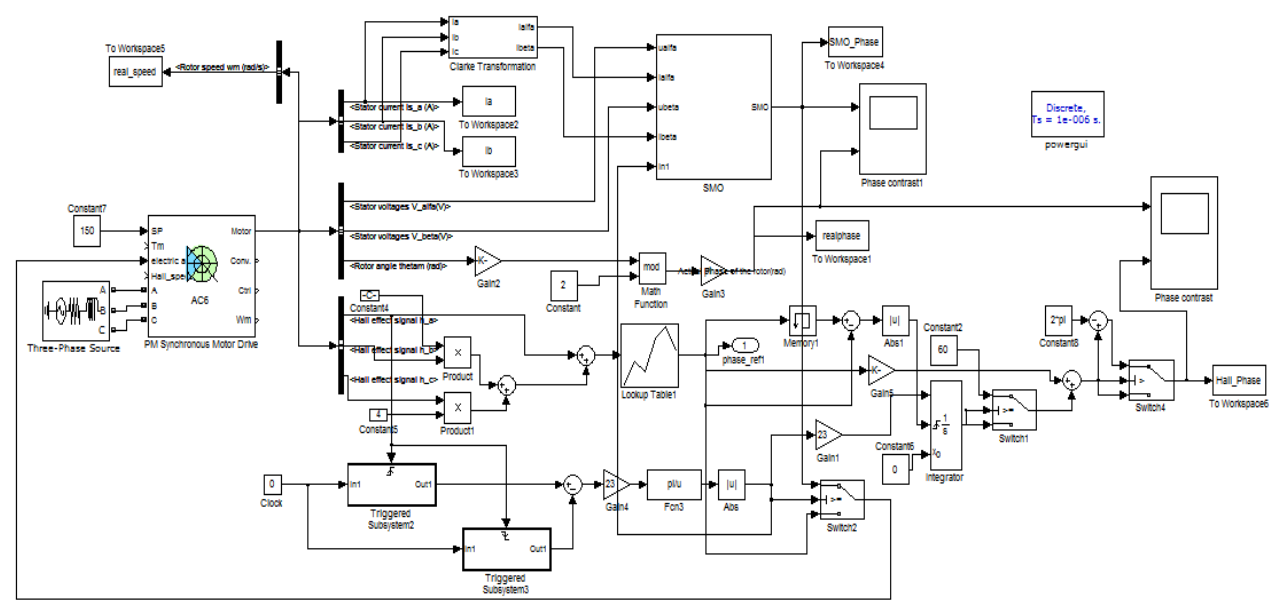

Fig. 4. Simulation model of the PMSM drive system.

The Starting State. Fig. 5 shows the phase estimation result when the PMSM is started by three Hall sensors. The estimated phase has some delay and ripple at first but the PMSM is started successfully finally.

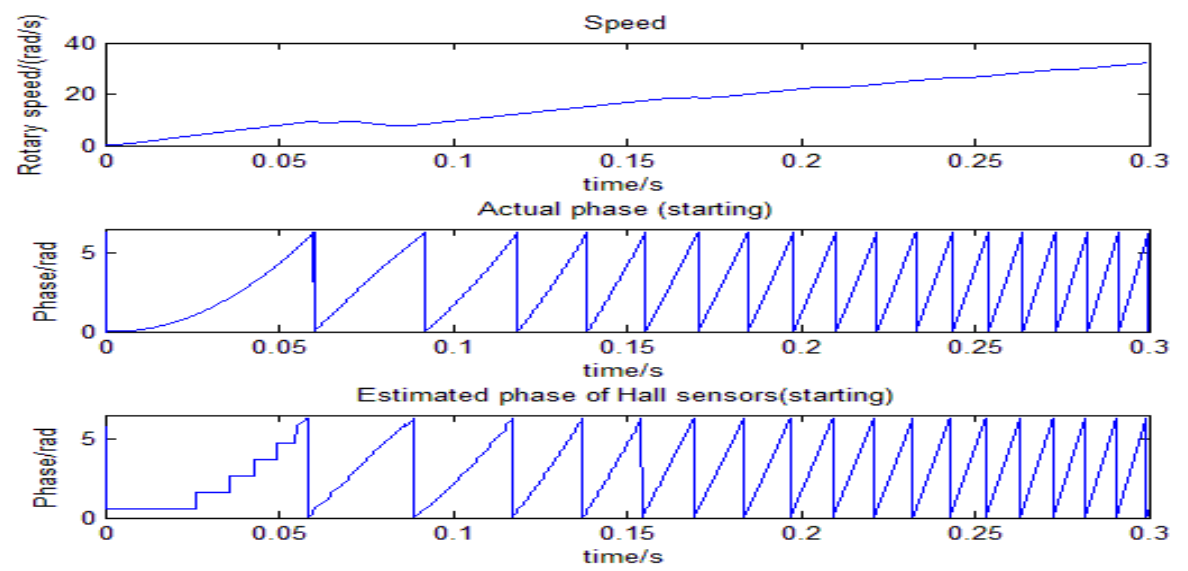

Fig. 5. The rotor phase when the PMSM is started by the Hall sensors.

Fig. 6 shows the starting process by the SMO under closed-loop control condition. It fails because that the estimated speed has lots of noise under the starting procedure and this noise will paralyze the control system. The PMSM is vibrating and loss control under starting state.

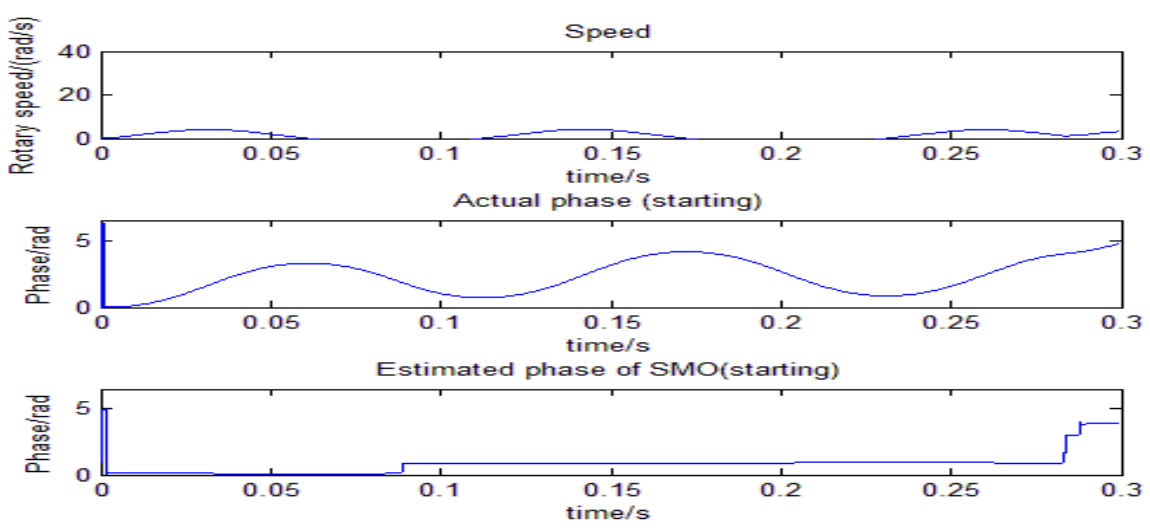

Fig. 6. The rotor phase when the PMSM is started by SMO. 
The Steady State. Fig. 7 and Fugure. 8 give the control results by Hall sensors under steady state. These three Hall sensors estimate rotor phase accurately and no ripple components occur. The phase currents are regular and no deformity found.

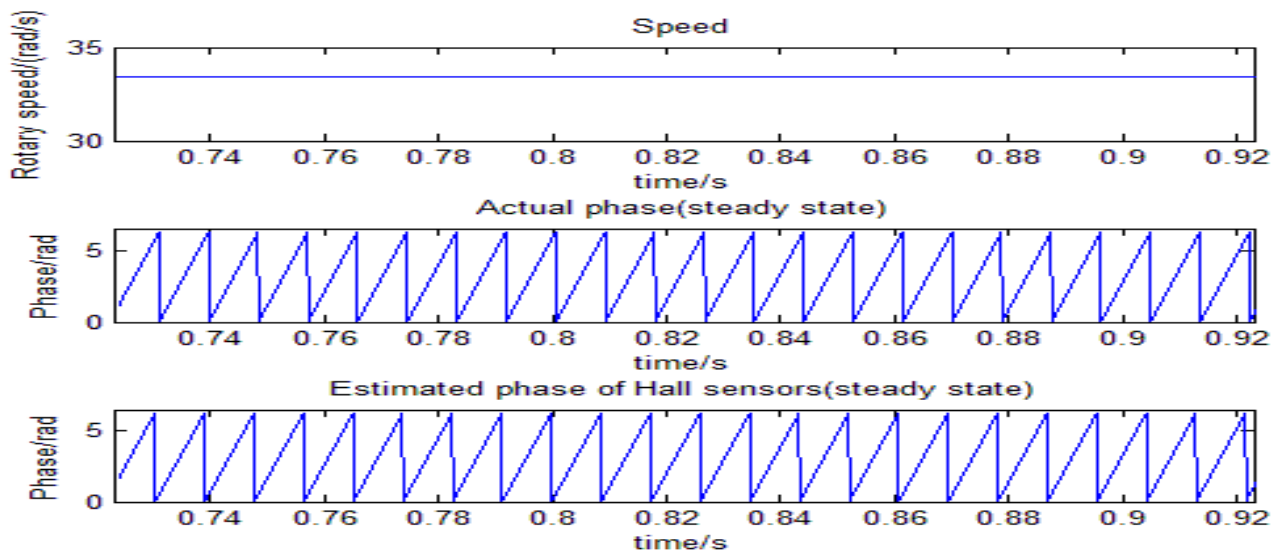

Fig. 7. The estimated phase controlled by Hall sensors under steady state.

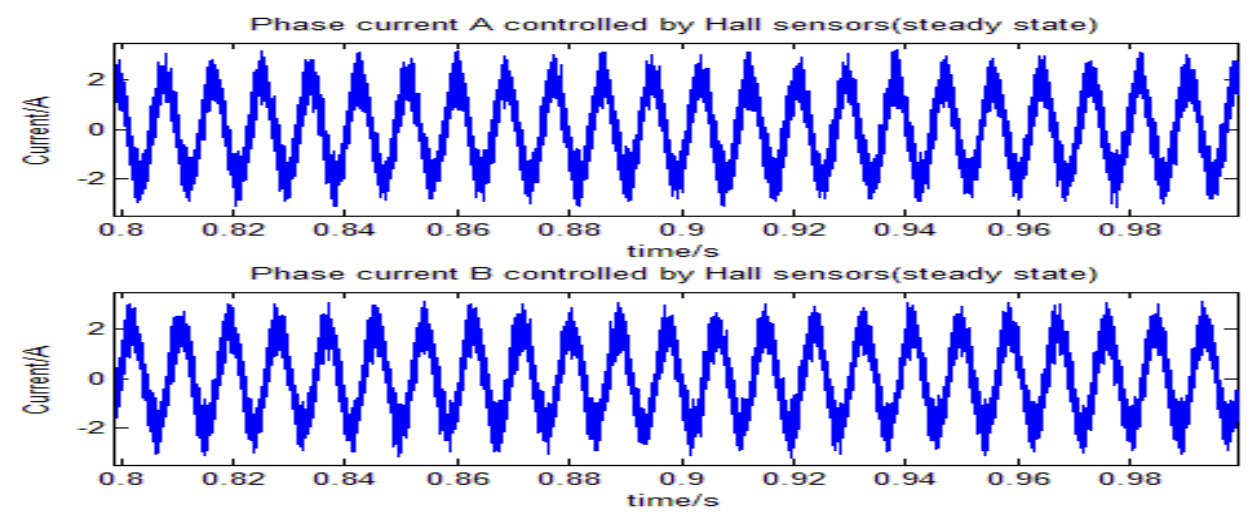

Fig. 8. Phase currents when the PMSM is controlled by Hall sensors under steady state.

Fig. 9 shows the phase estimation result when the PMSM is controlld by SMO under steady state. Contrasted with Fig. 7, it is obvious that the phase estimation result of SMO has some vibration components which is caused by the SMO algorithm and this has been expounded in section II.
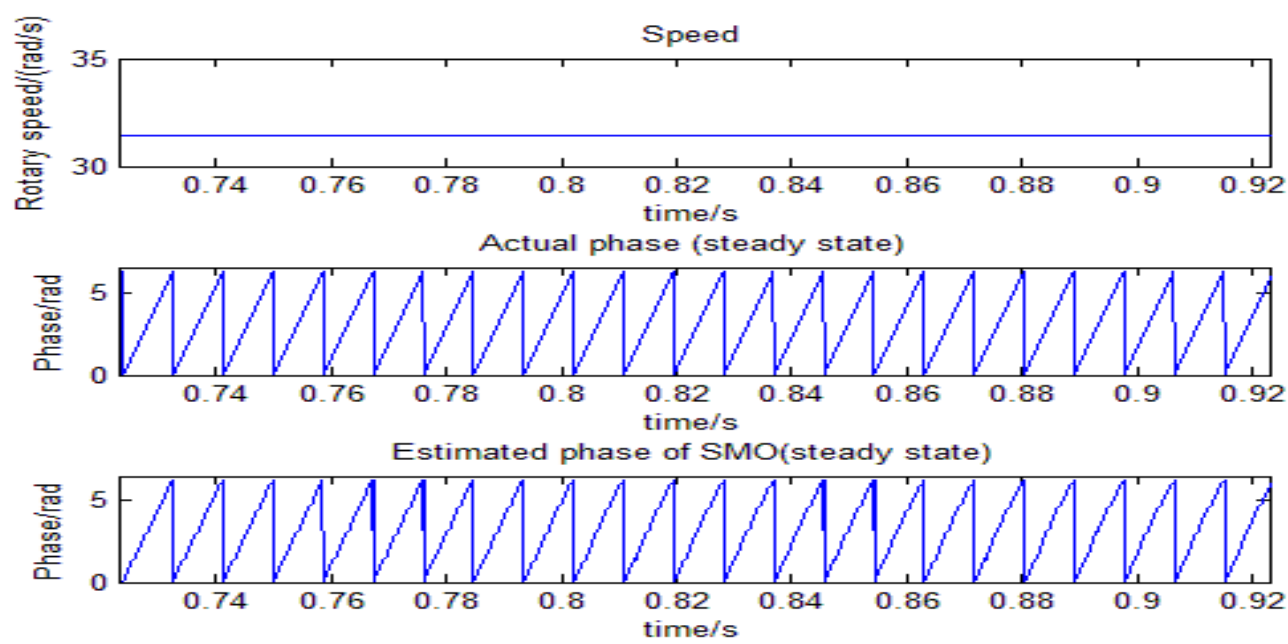

Fig. 9. The estimated phase when the PMSM is controlled by SMO under steady state. 
Fig. 10 shows the phase currents when the PMSM is controlld by SMO under steady state and these two currents have more noise contrasted with Fig. 8. The additional noise is caused by the vibration of estimated phase shown in Fig. 9.

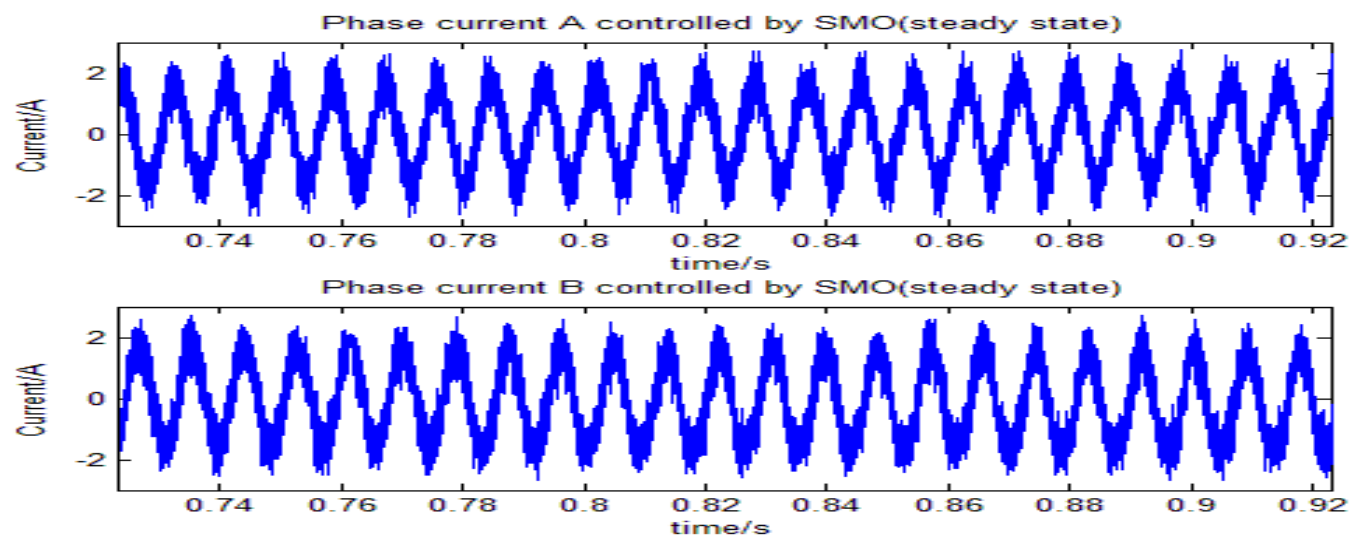

Fig. 10. Phase currents when the PMSM is controlled by SMO under steady state.

The Speed Fluctuation State. In actual application, the rotor speed of PMSM may fluctuate when the load or road condition changes. The fluctuating speed will bring some errors to the phase estimation of both SMO and Hall sensors. The phase estimation result of Hall sensors under speed fluctuation state is illustrated by Fig. 11 and some deformities can be found when contrasted with Fig. 7. The deformities are caused by the speed prediction error and delay which is illustrated in section III. Fig. 12 shows the phase currents when the PMSM is controlled by Hall sensors under speed fluctuation state and some deformity parts are found when contrasted with Fig. 8.

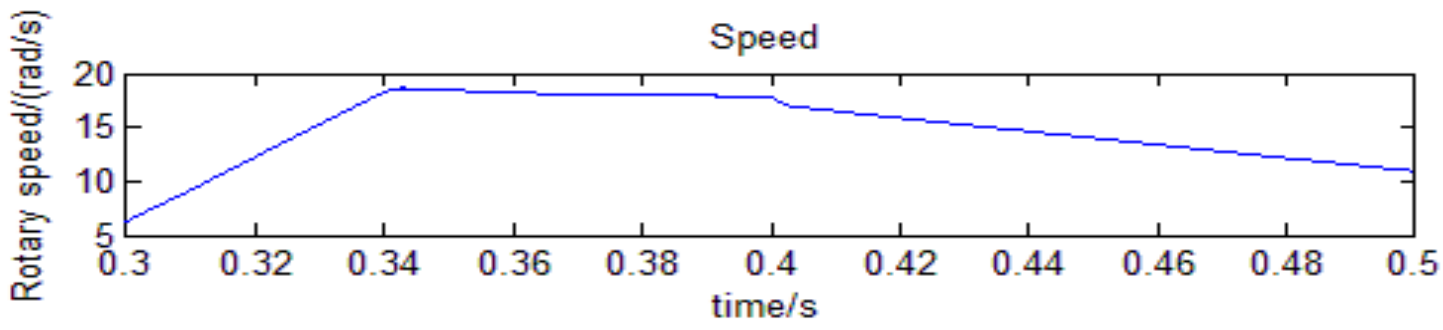

Actual phase(speed fluctuation state)

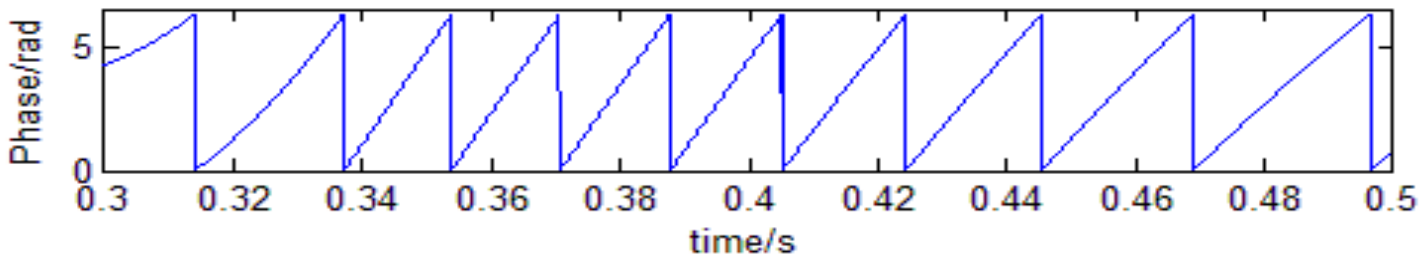

Estimated phase of Hall sensors(speed fluctuation state)

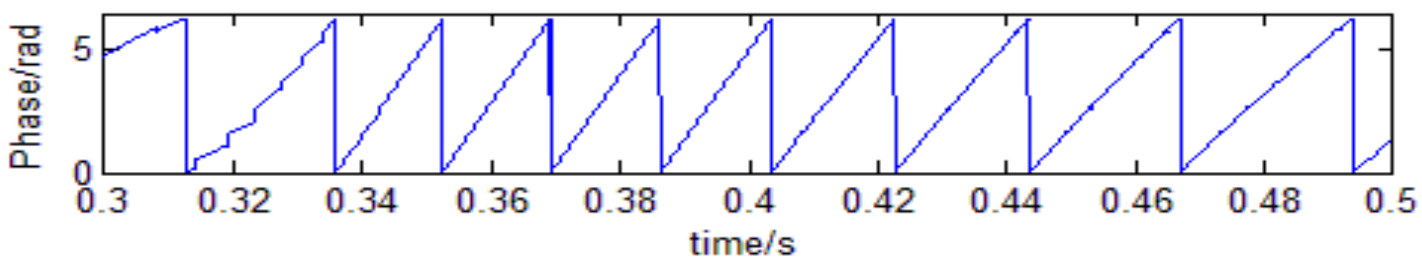

Fig. 11. The estimated phase when the PMSM is controlled by Hall sensors under speed fluctuation state. 

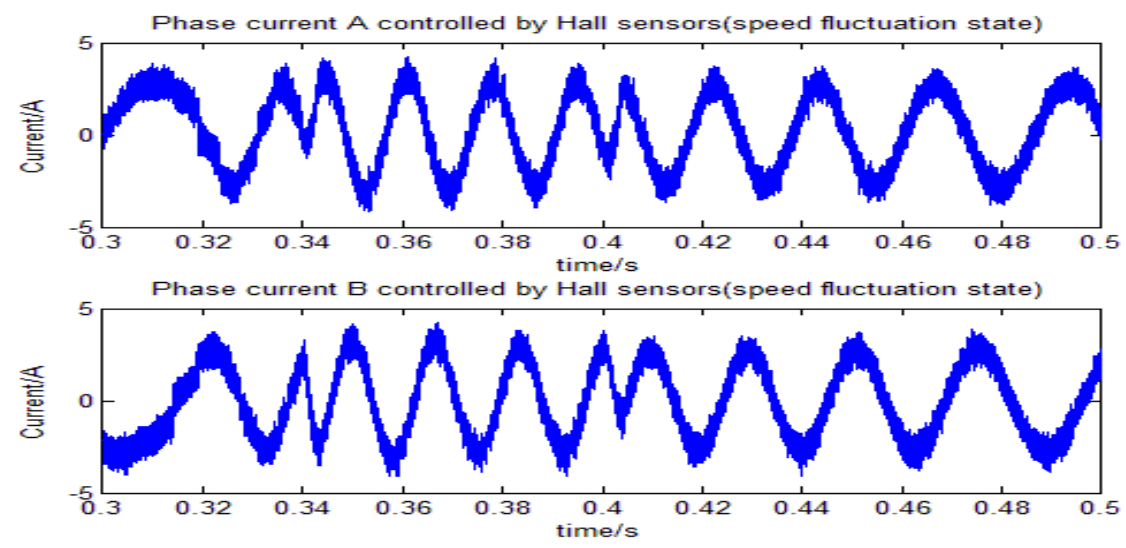

Fig. 12. Phase currents when the PMSM is controlled by Hall sensors under speed fluctuation state.

Fig. 13 shows the phase estimation result when the PMSM is controlled by SMO under speed fluctuation state, and no obvious additional vibration components occur when contrasted to Fig. 9. Fig. 14 give the simulation results of Phase currents and some deformities occur when contrasted with Fig. 10. Contrasting Fig. 14 with Fig. 12, no obvious difference is found.

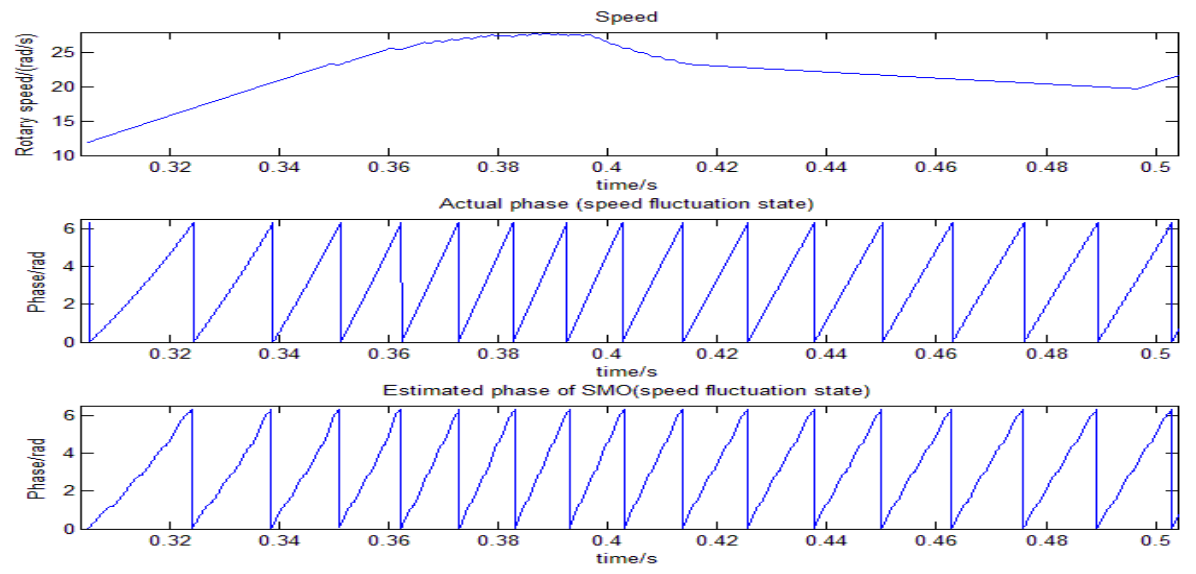

Fig. 13. The estimated phase when the PMSM is controlled by SMO under speed fluctuation state.
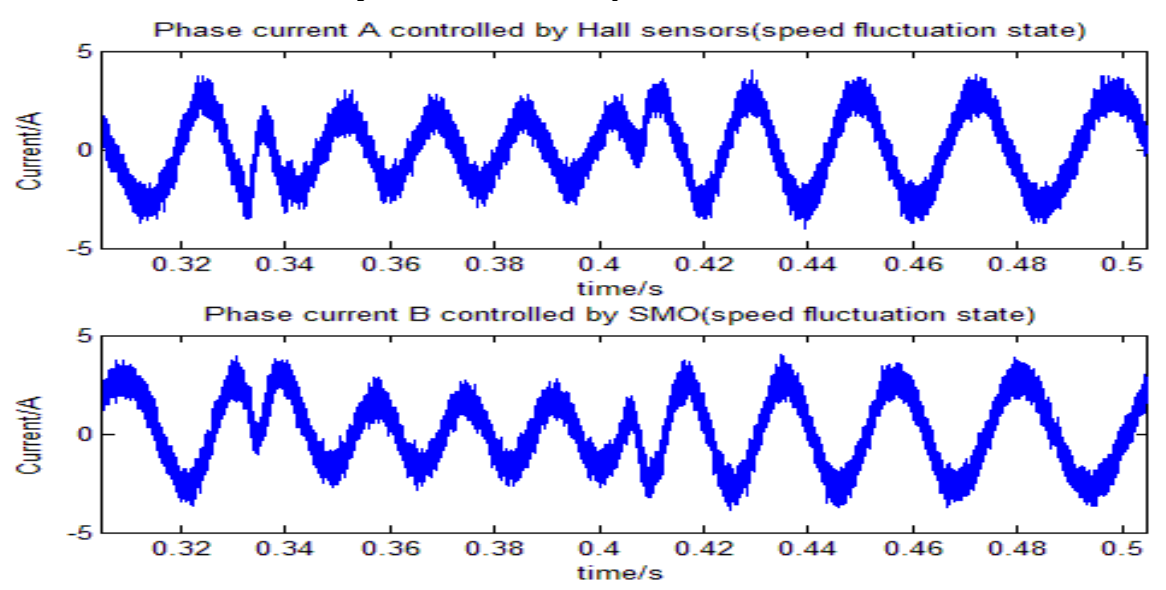

Fig. 14. Phase currents when the PMSM is controlled by SMO under speed fluctuation state. 


\section{Summary}

1) The PMSM can be started by Hall sensors whether the SMO cannot fulfil this task. This performance is essential to the actual application.

2) The control effect of Hall sensors under steady condition is better than the SMO. The phase estimation result and phase currents of SMO have some distinct vibration components which donot occur when the PMSM is controlled by Hall sensors.

3) The control effect of Hall sensors under speed fluctuation state has some distinct deformities which donot occur under steady state. The estimated phase of SMO under speed fluctuation state has no obvious differences with the result under steady state but the phase currents of SMO have some deformities.

4) The Hall senors have a better comprehensive performance than the SMO while the SMO has a better robustness to speed fluctuation and load change. The Hall sensors have a wide range of use in order to achieve a satisfied control effect. But taking the cost, reliability and encumbrance into account, the SMO also has some irreplaceable advantages and lots of applications.

\section{Acknowledgements}

Supported by the MOST (Ministry of Science and Technology) of China under the contract of No. 2010DFA72760 and No. 2012DFA81190, and the Tsinghua University Initiative Scientific Research Program (Grand No. 2010THZ08) is greatly acknowledged.

\section{References}

[1] Y. Honda, T. Nakamura, T. Higaki and Y. Takeda: submitted to IEEE-IAS Annual Meeting (1997).

[2] A. Benchaib, S. Poullain, J. L. Thomas and J.C. Alacoque: submitted to IEEE International Electrical Ma-chines and Drives Conference (2003).

[3] J. Simanek, J. Novak, O. Cerny and R. Dolecek: submitted to IEEE International Symposium on Industrial Electronics (2008).

[4] H. Kim, S. Yi, N. Kim and R. D. Lorenz: submitted to IEEE IAS Annual Meeting (2005).

[5] Alessandro Lidozzi, Luca Solero, Fabio Crescimbini and Augusto Di Napoli: submitted to IEEE Transaction On Power Electronics (2007).

[6] F.Parasiliti: submitted to International Symposium on Industrial Electronics (1997).

[7] J.-I. Ha, K. Ide, T. Sawa and S.-K. Sul: submitted to IEEE Transactions on Industry Applications (2003).

[8] Vadim Utkin, Sliding mode control in electromechanical systems, first ed., Taylor, Francis, 1999.

[9] Takeshi Furuhashi: submitted to IEEE Trans. Ind. Electronics (1992).

[10] K. Kang, J. Kim, K.-B. Hwang, and K.-H. Kim: submitted to IEEE APEC (2004). 